\title{
Research on the Coupling Relationship between Market Risk and Credit Risk in Commercial Banks
}

\author{
Jie $\mathrm{Su}^{1}$, Tian $\mathrm{Li}^{1}$ and $\mathrm{Xin} \mathrm{Ni}^{2}$ \\ ${ }^{1}$ Central University of Finance and Economics, School of Management Science and Engineering, 100081 Beijing, China \\ ${ }^{2}$ United Credit Ratings Co., Ltd., 100022 Beijing, China
}

\begin{abstract}
With the complexity and diversity of business development, commercial banks gradually put more focus on how to improve the accuracy of risk measurement. In this essay, we first defined the basic market risk and credit risk indexes by the use of the financial data of the target bank. Then, we built the Copula Model through Monte Carlo simulation techniques. We finally built the Copula- $\mathrm{VaR}$ measurement model which revealed the relationship between the two types of risks.
\end{abstract}

\section{Introduction}

With the increasing complexity and diversity of business development, commercial banks gradually put more focus on how to improve the accuracy of risk measurement.

Alexander et al. constructed the joint distribution of banks' market risk and credit risk by applying common risk factors [1]. Dimakos et al. estimated the total loss distribution of a financial institution by constructing an econometric model and employing Monte Carlo simulation method, which aggregated different relevant risks [2]. As the Copula function proposed by Sklar in 1959 can flexibly measure the degree of dependence between random variables and has good structure characteristics [3], Scholars like Straumann have applied it to research on financial risk measurement [4]. Rosenberg used the normal Copula function and t-Copula function to calculate the aggregating risk of market risk, operational risk, and credit risk of commercial banks [5]. Yao et al. utilized the peak value method and the conditional valueat-risk (CVaR) method, and adopted the Copula model to numerically simulate the expected and unexpected losses of operational risk in 14 commercial banks in China [6]. Hsu built extreme value Copula function, which solved the deviation problem caused by skewness and thick tails in the distribution which emerged when using the traditional method of calculating value at risk (VaR) [7].

On the other hand, with the rapid development of cross-disciplinary techniques and methods, the coupling theory, as an effective method in natural science, has been introduced into sociology studies. Weick is the first one who employed the coupled theory to explain economic and social problems. By applying the loose coupling theory, he obtained independent but related relationship between institutions relevant to schools [8]. Xu Taoguo demonstrated the feasibility of coupling risk management on interest rate risk and credit risk from both theoretical and empirical perspectives. Then he used Monte Carlo simulation method and Copula function to establish an evaluation model of the coupling risk [9]. Yan Chao established a coupling degree model based on the coupling theory to analyze profitability and risk management ability of commercial banks [10]. He Xubiao constructed a VaR coupling model to conduct a coupling study of listed companies' credit risk and interest rate risk and measure the aggregating risk of assets [11].

Based on the main indicators of commercial bank risk management, this paper presents a research focusing on the coupling between market risk and credit risk of commercial banks. First of all, we selected appropriate indicators to reflect the risk return rate of China Merchants Bank's market risk and credit risk. Then, by establishing the Copula model, the correlation between the two types of risks and the joint distribution function were obtained. Based on this, by combining the VaR method and Monte Carlo simulation method, the VaR after coupling could be obtained, while by setting different proportions of risk assets, the coupling VaR values under different asset proportions could be obtained. Finally, the optimal risky portfolio was determined.

\section{Selection of market risk (MR) and credit risk (CR) indicators in commercial banks}

In this paper, China Merchants Bank was selected as a research sample and its 35 quarterly financial statement data from 2007 to September 2015[12] was integrated to acquire market risk and credit risk return rate indicators.

\subsection{Determination of credit risk return rate}


In actual banking business, the parameter related to credit risk is the interest income from the loan. In this paper, "Interest Income" in the income statement is used to represent the return for calculating the credit risk return rate. At the same time, "Loans and Payments on Behalf" in the balance sheet is used as the measurement indicator of credit risk asset amount. As a result, the credit risk return rate can be described as follows:

$$
\mathrm{Crr}=\frac{\text { interest income }}{\text { loans and payments on behalf }}
$$

where $C r r$ is the credit risk return rate.

\subsection{Determination of market risk return rate}

Revenue items related to market risk mainly include "Net Interest Income", "Handling Charges and Commissions Income", "Gain or Loss on Foreign Exchange Transactions", "Net Investment Income" and "Gain or Loss from Changes in Fair Values". When determining the market risk return rate, we add these five items to calculate market risk income.

When it comes to market risk asset amount, there are six items should be taken into consideration, including "Financial Assets Held for Trading", "Derivative Financial Assets", "Redemptory Monetary Capital for Sale", "Financial Assets Available for Sale", "Held-ToMaturity investments", and "Long-term Equity Investment". The sum of these six items represents market risk assets.

The formula of calculating market risk return rate is as follows:

$$
M r r=\frac{\text { market risk income }}{\text { market risk assets }}
$$

where Mrr is the market risk return rate.

\section{Determination of the Copula function}

In order to construct the Copula function of market risk return rate and credit risk return rate, the first step is to determine the marginal distribution of two variables. According to the definition of market risk return rate and credit risk return rate in the previous section, we extract the sample observation data of market risk return rate and credit risk return rate from the quarterly financial data of China Merchants Bank from 2007 to 2014. Next, the output of the basic descriptive statistical characteristics of the two variables and the correlation test results are obtained by using Eviews.

\subsection{Normality test}

In the first place, as is shown by kurtosis and skewness information of the sample data, the sample data represents features of leptokurtosis and slightly fat-tail. Based on this observation, the normality of the two variables is further tested.

Since the amount of sample data selected in this paper is not sufficient, we conduct three normality tests for two types of risk-return sample data at the same time, including Kolmogorov-Smith test, Jarque-Bera test and Lilliefors test. The first two tests have good results when tested on small samples. By arriving at the p-values for the three types of tests, whether the variables have normality is judged. The result is shown in Table 1.

Table 1. Normality test of market risk return rate (MAR) and credit risk return rate (CRE).

\begin{tabular}{|c|c|c|}
\hline \multirow{2}{*}{ P-value } & \multicolumn{2}{|c|}{ Risk Return Rate } \\
\cline { 2 - 3 } & $\boldsymbol{M A R}$ & $\boldsymbol{C R E}$ \\
\hline Kolmogorov-Smith test & 0.1753 & 0.001 \\
\hline Jarque-Bera test & 0.5379 & 0.3216 \\
\hline Lilliefors test & 0.1247 & 0.0287 \\
\hline
\end{tabular}

As the results of K-S test and Lilliefors test reflect, we cannot reject the null hypothesis that the variable of the market risk return rate obeys the normal distribution. On the other hand, as for the test results of the credit risk return rate, the p-values are too small, thus the null hypothesis that the sample variable obeys the normal distribution should be rejected. The results of Jarque-Bera test show that both null hypotheses that risk return rate is normally distributed should not be rejected.

\subsection{Determining the sample marginal distribution}

When employing the Copula model, due to the inconsistency of the normality test results, the kernel density estimation (KDE), as a non-parametric method, is used to estimate the sample distribution. The empirical distribution function and the nuclear density distribution estimation are obtained (see Figure 1 and Figure 2).

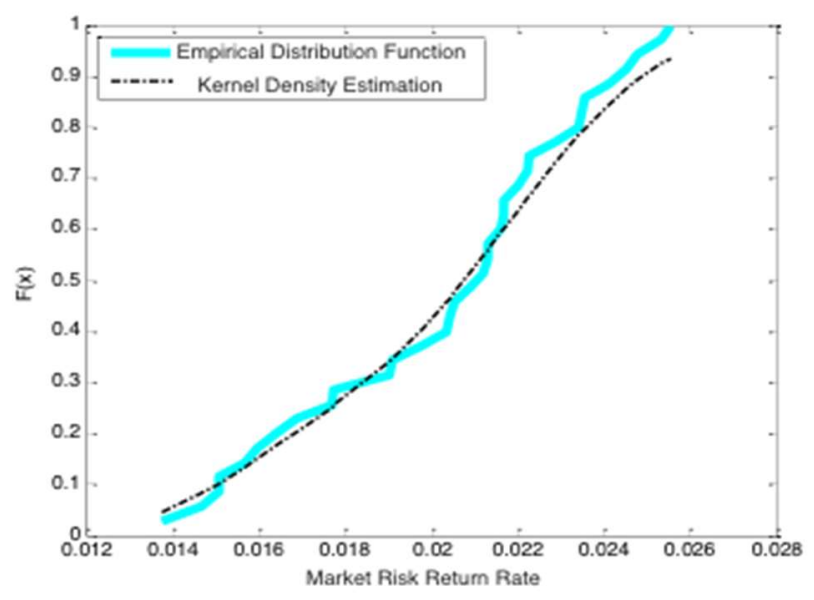

Figure 1. Empirical distribution function and kernel distribution estimation of market risk return rate. 


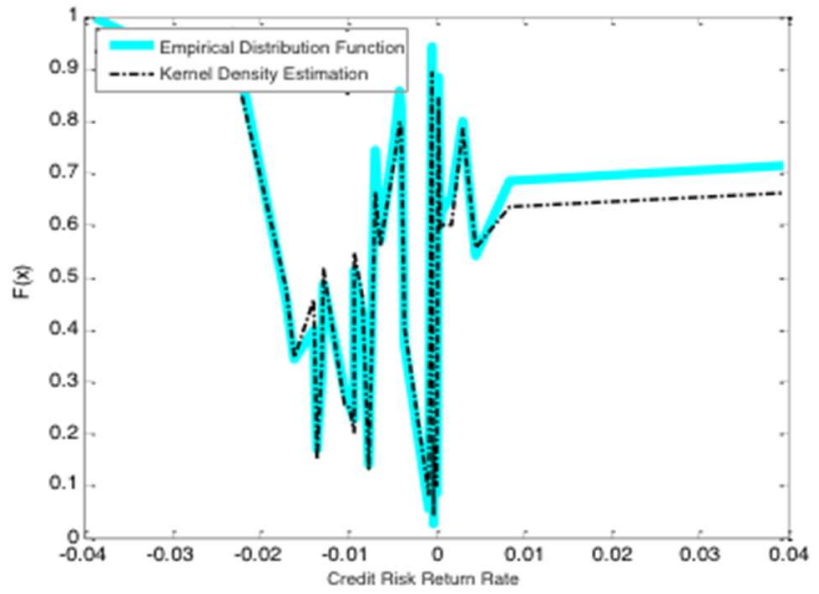

Figure 2. Empirical distribution function and kernel distribution estimation of credit risk return rate.

As Figure. 1 and Figure. 2 depict, the kernel distribution estimation and empirical distribution functions of the two types of risk return rates can fit well. Therefore, the kernel distribution function is chosen as the marginal distribution function of the market risk return rate and the credit risk return rate.

\subsection{Parameter estimation of Copula function}

In order to form appropriate Copula connection functions which can adapt to both marginal distribution functions and link them, we choose two kinds of Copula functions with elliptical dependence structures (the binary normal Copula and t-Copula) as the binary joint distribution functions of the two kinds of risk return rates, the symmetrical tail of the joint density function ensures the rationality of this selection. By using the semi-parametric estimation method, the relevant parameter estimates for the two types of Copula functions are obtained. The results are shown in Table 2:

Table 2. Parameter estimation of two Copula models.

\begin{tabular}{|c|c|c|}
\hline \multirow{2}{*}{} & \multicolumn{2}{|c|}{ Parameter Estimation } \\
\cline { 2 - 3 } & $\begin{array}{c}\text { Correlation } \\
\text { coefficient }\end{array}$ & $\begin{array}{c}\text { Degree of } \\
\text { freedom }\end{array}$ \\
\hline $\begin{array}{c}\text { Normal Copula } \\
\text { model }\end{array}$ & -0.1415 & \\
\hline t-Copula model & -0.0241 & 3.4511 \\
\hline
\end{tabular}

Thus, the binary normal Copula function of the two types of risk returns is:

$$
C^{G a}(\hat{u}, \hat{v})=\int_{-\infty}^{\emptyset^{I}(\hat{u})} \int_{-\infty}^{\varnothing^{I}(\hat{v})} \frac{\exp \left[-\frac{s^{2}-2 \times(-0.1415) s t+t^{2}}{2 \times\left(I-(-0.1415)^{2}\right)}\right]}{2 \pi \sqrt{1-(-0.1415)^{2}}} d s d t
$$

The degree of freedom of the binary t-Copula is about 3. When inputting linear correlation parameters and degrees of freedom into the distribution function, we can get the binary t-Copula function of two risk return rates:

$$
C^{t}(\hat{u}, \hat{v})=\int_{-\infty}^{t_{3}^{-1}(\hat{u})} \int_{-\infty}^{t_{3}^{-l}(\hat{v})} \frac{\exp \left[-\frac{s^{2}-2 \times(-0.0241) s t+t^{2}}{2 \times\left(I-(-0.0241)^{2}\right)}\right]}{2 \pi \sqrt{1-(-0.0241)^{2}}} d s d t
$$

\section{Evaluation of Copula model}

To calculate the coupling VaR, we shall select the Copula model which can fit the empirical function best, so we need to evaluate the fitting effect of the two models first. With the introduction of the empirical distribution functions of the sample, the square Euclidean distances between the distribution function and the empirical distribution function under the two models are calculated. Therefore, the validity of the Copula model is examined.

\subsection{Defining the empirical Copula function}

Let $\left(x_{i}, y_{i}\right), i=1,2, \cdots, n$ be samples from a two-dimensional population $(X, Y)$, and denote empirical distributions of $X$ and $Y$ as $F_{n}(x)$ and $G_{n}(y)$. The empirical Copula function is

$$
\widehat{C}_{n}(u, v)=\frac{1}{n} \sum_{i=1}^{n} I_{\left[F_{n}\left(x_{i}\right) \leq n\right]} I_{\left[G_{n}\left(y_{i}\right) \leq n\right]}, u, v \in[0,1]
$$

where $I_{[\cdot]}$ is a characteristic function, if $F_{n}\left(x_{i}\right) \leq u$, $I_{\left[F_{n}\left(x_{i}\right) \leq n\right]}=1$, otherwise $I_{\left[F_{n}\left(x_{i}\right) \leq n\right]}=0$.

After obtaining the empirical Copula function $\widehat{C}_{n}(u, v)$, we can calculate the squared Euclidean distance between the binary normal Copula model and the t-Copula model. The formulas are as follows:

$$
\begin{gathered}
d_{G a}^{2}=\sum_{i=1}^{n}\left|\widehat{C}_{n}\left(u_{i}, v_{i}\right)-\widehat{C}^{G a}\left(u_{i}, v_{i}\right)\right|^{2} \\
d_{t}^{2}=\sum_{i=1}^{n}\left|\widehat{C}_{n}\left(u_{i}, v_{i}\right)-\widehat{C}^{t}\left(u_{i}, v_{i}\right)\right|^{2}
\end{gathered}
$$

where $u_{i}=F_{n}\left(x_{i}\right), v_{i}=G_{n}(i=1,2, \cdots, n), d_{G a}^{2}$ and $d_{t}^{2}$ represent fitting effect of two Copula models. If $d_{G a}^{2} \leq d_{t}^{2}$, then the binary normal Copula model can fit the original sample data better. If $d_{G a}^{2} \geq d_{t}^{2}$, then the binary t-Copula model shows a better fitting effect on the original data.

\subsection{Calculating the squared Euclidean distances between the two models and the empirical Copula}

After calculating the squared Euclidean distances between the two models and the empirical Copula function, the square Euclidean distances of the binary normal Copula model and the binary t-Copula model are acquired as 0.0659 and 0.0497.Apparently, the squared Euclidean distance of the binary t-Copula model and the empirical Copula function $\left(d_{t}^{2}\right)$ is smaller than that of the binary normal Copula model and the empirical Copula function $\left(d_{G a}^{2}\right)$. Evidently, under the criterion of squared Euclidean distance, the binary t-Copula model can fit the sample data of market risk return rate and credit risk return rate better.

\section{VaR calculation}

\subsection{Estimation of rank correlation coefficients}


By defining the Copula function, we have obtained the linear correlation parameters of the binary normal Copula model and the t-Copula model. In this section, we examine the correlation between the market risk return rate and the credit risk return rate by finding the rank correlation coefficient of the binary normal Copula model and the binary t-Copula model.

As for the selection of rank correlation coefficient, we use Kendall rank correlation coefficient and Spearman rank correlation coefficient as the basis for detecting correlation. The rank correlation coefficients of the binary normal Copula model are denoted as Kendall_norm and Spearman_norm, respectively, while the rank correlation coefficients of the binary t-Copula model are denoted as Kendall $t$ and Spearman $t$. By using MATLAB, the estimated correlation coefficients of the two models can be attained.

Table 3. Rank correlation coefficients for binary normal Copula model and binary t-Copula model.

\begin{tabular}{|c|c|c|}
\hline \multirow{2}{*}{$\begin{array}{c}\text { Rank Correlation } \\
\text { Coefficient }\end{array}$} & \multicolumn{2}{|c|}{ Model } \\
\cline { 2 - 3 } & $\begin{array}{c}\text { Binary Normal } \\
\text { Copula }\end{array}$ & Binary t-Copula \\
\hline $\begin{array}{c}\text { Kendall rank } \\
\text { correlation coefficient }\end{array}$ & -0.0904 & -0.0153 \\
\hline $\begin{array}{c}\text { Spearman rank } \\
\text { correlation coefficient }\end{array}$ & -0.1352 & -0.0230 \\
\hline
\end{tabular}

From the estimated rank correlation coefficient values, it is obvious that the rank correlation coefficients between variables for the two Copula models are all negative, which means that there is a negative correlation between market risk return rate and credit risk return rate.

Besides, we also directly apply the raw sample yield data into the correlation test to obtain the Kendall rank correlation coefficient and the Spearman rank correlation coefficient of the sample. The validity of the rank correlation coefficients is judged by comparing the rank correlation coefficients of the two models with the rank correlation coefficients for the original sample data. By directly using the sample data, the Kendall rank correlation coefficient and Spearman rank correlation coefficient for the two risk returns are obtained as -0.0185 and -0.0238 . Therefore, Kendall_t and Spearman_t are closest to the correlation coefficients of the original data. So binary tCopula is the better choice.

\subsection{Calculating and comparing VaR}

Based on the parameter estimates and the fitted binary tCopula function, the Monte Carlo simulation method is used in this section to calculate the coupled risk value of market risk return and credit risk return. In New Basel Accord, it is proposed that when calculating VaR value for the market risk, confidence level of $99 \%$ in one-tailed test needs to be met. In the estimation of VaR coupling risk, we also adopt this confidence level and perform 5000 simulations.

Firstly, in the calculation of VaR, we set different proportions of risk assets and calculate $\mathrm{VaR}$ of the asset portfolios. When simulating the coupled VaR, we first estimate the specific parameters of the $\mathrm{t}$-Copula function and get the t-Copula binary distribution function. Then we generate two independent random numbers ( $u$ and $v$ ) which obey 0-1 distribution for 5000 times. Thus, according to the number pairs acquired, the corresponding market risk and credit risk can be obtained. Next, we calculate the portfolio returns under different asset portfolios and calculate the coupled $\mathrm{VaR}$ at a $99 \%$ confidence level. Besides, in order to illustrate the coupling relationship between commercial banks' market risk and credit risk, we compare the calculation result with the VaR values calculated by the traditional variancecovariance method.

Table 4. Copula-VaR values at different risk asset proportions (confidence level is $99 \%$ ).

\begin{tabular}{|c|c|c|}
\hline \multirow{2}{*}{$\begin{array}{c}\text { Risk Asset } \\
\text { Proportion }^{\text {a }}\end{array}$} & \multicolumn{2}{|c|}{ Values } \\
\cline { 2 - 3 } Copula-VaR & VaR \\
\hline $0: 10$ & 0.0334 & 0.0663 \\
\hline $1: 9$ & 0.0309 & 0.0601 \\
\hline $2: 8$ & 0.0283 & 0.0544 \\
\hline $3: 7$ & 0.0261 & 0.0496 \\
\hline $4: 6$ & 0.0239 & 0.0451 \\
\hline $5: 5$ & 0.0225 & 0.0434 \\
\hline $6: 4$ & 0.0212 & 0.0423 \\
\hline $7: 3$ & 0.0207 & 0.0438 \\
\hline $8: 2$ & 0.0204 & 0.0469 \\
\hline $9: 1$ & 0.0203 & 0.0512 \\
\hline $10: 0$ & 0.0201 & 0.0560 \\
\hline
\end{tabular}

a. The "risk assets proportion" in the table is "market risk assets: credit risk assets"

As is shown in the Table 4, the VaR of the aggregation of market risk and credit risk under the Copula model is lower than the VaR calculated under the traditional method. Obviously, the traditional method of calculating $\mathrm{VaR}$ is likely to overestimate the systematic risk. As a result, commercial banks may increase the relevant economic capital and loan loss reserve ratio in the supervision of internal risks, which will reduce the efficiency of the use of funds and lose revenue.

Moreover, there is a non-linear negative correlation between the market risk return and the credit risk return of commercial banks. By calculating the VaR values under different risk asset proportions, it is observed with the increase of market risk, the VaR of the commercial bank decrease slightly, and when the credit risk occurs, it will most likely cause greater losses.

\section{Conclusion}

In this paper, we mainly focus on the coupling of market risk and credit risk in commercial banks. There are 2 main parts: the research on whether the coupling between the two exists, and the quantitative analysis of the coupling between the two risks. Firstly, in the coupling study, the Copula model is introduced to link the commercial bank's 
market risk with the credit risk and conduct the study on correlation to verify the correlation between the two risks. The results show that there is a negative correlation between market risk and credit risk in commercial banks. Then, we compare the VaR values under the traditional VaR method and the Copula model. By comparing calculating results the $\mathrm{VaR}$ of different risky asset proportions under different models, it is found the traditional variance-covariance method is likely to overestimate the VaR of the integrated risk, thus the accuracy of $\mathrm{VaR}$ calculation can be improved by employing Copula model. By using Copula model in the actual risk management operations of commercial banks, economic capital will be reduced and the capital efficiency will be improved. In addition, it is found that the VaR of integrated risk will decrease as the proportion of market risk assets increases.

There are also some limitations in the study of this paper, which requires in-depth digging and improvement: First, the amount of sample data in this paper is not sufficient. Due to the limited listing period for domestic banks, the data extracted are mainly unaudited quarterly financial statements to ensure the accuracy and sufficiency of sample data. Second, because of the distribution characteristics of sample data, in this paper, we only select two Copula models for modeling analysis. In future studies, Other scholars might combine more methods and introduce more conditional Copula functions to perform dynamic correlation analysis of risks.

\section{Acknowledgment}

This work is supported by Program for Innovation Research in Central University of Finance and Economics. We acknowledge the support of this excellent program in CUFE.

\section{References}

1. C. Alexander, J. Pézier, "On the aggregation of market and credit risks", ISMA Centre Discussion
Papers in Finance, No. 2003-13, University of Reading, 2003.

2. K. Aas, X. K. Dimakos, A. Øksendal, "Risk capital aggregation”, Risk Management, 2007, 9, pp.82-107.

3. M. Sklar, "Fonctions de Répartition À N Dimensions Et Leurs Marges", Publications de Institut de Statistque Universite de Paris, 1959, 8, pp.229-231.

4. P. Embrechts, A. J. McNeil, D. Straumann, "Correlation and dependence in risk management: properties and pitfalls", Risk Management Value at Risk \& Beyond, 2000, pp.176-223.

5. J. V. Rosenberg, T. Schuermann, "A general approach to integrated risk management with skewed, fat-tailed Risks", Journal of Financial Economics. 2006, 79 (3), pp.569-614.

6. F. G. Yao, H. M. Wen, J. Q. Luan, "CVaR measurement and operational risk management in commercial banks based on peak value method of extreme theory", Mathematical and Computer Modelling, 2013, 58 (1-2), pp.15-27.

7. C. P. Hsu, C. W. Huang, W J P. Chiou, "Effectiveness of copula-extreme value theory in estimating value-atrisk: empirical evidence from Asian emerging markets", Review of Quantitative Finance and Accounting, 2012, 39 (4), pp.447-468.

8. K. E. Weick, "Educational organization as loosely coupled systems", Administrative Science Quarterly, 1976, 21 (1): pp.1-19.

9. T. G. Xu, "The study of coupling interest rate risk and credit risk in bond investment", Wuhan: Huazhong University of Science and Technology, 2008. (In Chinese)

10. C. Yan, "Research on the coupling relationship between profitability and risk management capability of commercial banks", Financial Perspectives Journal. 2014, (12), pp.80-86. (In Chinese)

11. X. B. He, "Research on VaR model for risks' coupling theory and numerical simulation technology and its applications" Wuhan: Huazhong University of Science and Technology, 2006. (In Chinese)

12. China Merchants Bank annual report data is taken from wind, http://www.wind.com.cn/NewSite/data. html. 